\title{
Three-dimensional Ising model confined in low-porosity aerogels: A Monte Carlo study
}

\author{
Ricardo Paredes V. ${ }^{1,2}$ and Carlos Vásquez ${ }^{3}$ \\ ${ }^{1}$ Centro de Física, Instituto Venezolano de Investigaciones Científicas, Apartado 21827, Caracas 1020A, Venezuela \\ ${ }^{2}$ Technische Universiteit Delft, DelftChemTech, Particle Technology, Julianalaan 136, 2628 BL, Delft, The Netherlands \\ ${ }^{3}$ Departamento de Física, Universidad Simón Bolívar, Apartado 89000, Caracas 1080A, Venezuela \\ (Received 11 October 2005; revised manuscript received 7 June 2006; published 2 August 2006)
}

\begin{abstract}
The influence of correlated impurities on the critical behavior of the three-dimensional (3D) Ising model is studied using Monte Carlo simulations. Spins are confined into the pores of simulated aerogels (diffusionlimited cluster-cluster aggregation) in order to study the effect of quenched disorder on the critical behavior of this magnetic system. Finite-size scaling is used to estimate critical couplings and exponents. Long-range correlated disorder does not affect the critical behavior. Asymptotic exponents differ from those of the pure 3D Ising model, but it is impossible, with our precision, to distinguish them from the randomly diluted Ising model.
\end{abstract}

DOI: 10.1103/PhysRevB.74.054201

\section{INTRODUCTION}

The influence of quenched disorder on phase transitions has been studied for a long time now. In 1974, Harris ${ }^{1}$ established this famous criterion: uncorrelated disorder is not relevant, for a second-order phase transition, if the specific heat exponent is negative $(\alpha<0)$. The criterion was generalized by Weinrib and Halperin ${ }^{2}$ (WH) for any disorder distribution whose correlation function exhibits a power-law tail-i.e., $g(r) \sim r^{-a}$ as $r \rightarrow \infty$. Disorder is shown to be relevant in these cases:

$$
\begin{aligned}
& d \nu-2<0 \quad \text { if } a \geq d, \\
& a \nu-2<0 \quad \text { if } a<d,
\end{aligned}
$$

$d$ being the dimension and $\nu$ the correlation length exponent of the pure system. After Josephson hyperscaling $(2-d \nu$ $=\alpha)$ the Harris criterion is recovered in the short-range correlated (SRC) regime (1). In contrast, the long-range correlated (LRC) regime extends the criterion to systems satisfying condition (2), even if $\alpha<0$. This generalization explains why critical exponents for the superfluid (SF) transition of ${ }^{4} \mathrm{He}$ change when the fluid is confined in very light aerogels $\mathrm{s}^{3,4}$ and do not when confined in, for example, porous gold. ${ }^{5}$ Aerogels are fractal for several length scales, ${ }^{6}$ while porous gold has exponentially decaying correlations beyond the size of a typical pore. ${ }^{5}$ Nevertheless, the authors ${ }^{3,4}$ argued that the critical behavior of $\mathrm{SF}^{4} \mathrm{He}$ in aerogels yet poses intriguing questions to be solved.

For instance, light aerogels are fractal for several length scales, up to a certain value $\Lambda$ that depends on aerogel density. Beyond this length, the disordered structure becomes homogeneous, entering an uncorrelated regime. After the Harris criterion, as the SF correlation length $\xi$ gets larger than $\Lambda$, disorder should become irrelevant, because $\alpha \simeq$ -0.011 is negative for this system. Yoon et al. ${ }^{4}$ estimated that this crossover should appear at $t=\left|T-T_{c}\right| / T_{c} \simeq 10^{-4}$ but, although they approached $T_{c}$ as close as $t \simeq 10^{-5}$, no crossover to bulk exponents was observed. A different universality class was evident for the SF transition of ${ }^{4} \mathrm{He}$, when confined in aerogels. An explanation to these changes was given using
Monte Carlo (MC) simulations of the three-dimensional (3D) $X Y$ model, confined in aerogel-like structures. ${ }^{7}$ The SF transition belongs to the 3D $X Y$ universality class, and correlated disorder could be relevant provided that the WH condition (2) at $r \rightarrow \infty$ is fulfilled. Vásquez et al. ${ }^{7,8}$ showed that changes occur because of hidden LRC, inherent to the process of aerogel formation. Using simulated aerogels, made by diffusion-limited cluster-cluster aggregation ${ }^{9,10}$ (DLCA), the authors showed that different LRC subsets are physically well defined within the whole aerogel structure. Specifically, gelling clusters (to be defined later in this paper) are shown to be the relevant structures defining the critical behavior of the 3D $X Y$ model in DLCA aerogels. ${ }^{7}$

In this paper, we study the three-dimensional Ising (3DIS) model, in the presence of such aerogel-like structures. The pure 3DIS model has a positive specific heat exponent $(\alpha$ $\simeq 0.11$ ), so any type of disorder, correlated or not, will be relevant. If Ising spins are collocated in the pores of aerogels, criticality will be affected by LRC as well as by SRC disorder. Our main purpose is to elucidate which among these effects dominates the critical behavior of the 3DIS model in this case. Along this paper, we report the results of extensive MC simulations of the 3DIS model in the pores of DLCA aerogels at fixed porosity $\varphi=80 \%$, in order to clarify this point.

The rest of this paper is organized as follows: Section II is a brief review about diluted Ising systems studied in the past. Section III explains the model first, then the simulation procedure in detail, with a preamble on self-averaging, in order to validate our procedure; two methods to obtain accurate values of the critical coupling are presented at the end. Thermal and magnetic effective exponents are presented, and their asymptotic behaviors are discussed in Sec. IV. Finally, Sec. V presents some concluding remarks.

\section{ANTECEDENTS OF THE DILUTED 3DIS MODEL}

Many experimental, theoretical, and computational works have been done to date in order to study the critical behavior of the 3DIS model in the presence of quenched disorder. Most numerical and theoretical works address the random- 
diluted 3DIS (RDIS) model-i.e., the Ising model in the presence noncorrelated distributions of impurities. ${ }^{11-15}$

Apart, concerning disordered LRC structures, Ballesteros and Parisi ${ }^{16}$ simulated the 3DIS model, with dislocations represented by lines of impurities generated at random. Correlations for this type of disorder decay with an exponent $a$ $=2$. They obtain a correlation length exponent $\nu_{\mathrm{LRC}} \approx 1$ for the impure system, thus confirming the result of $\mathrm{WH}$, that this exponent should be $\nu^{2} \nu_{\mathrm{LRC}}=2 / a$. Marqués et al. ${ }^{17}$ also simulated a diluted 3DIS model, but with spins located on LRC sites. These sites were provided by a previous simulation of the pure 3DIS model; taking all sites from the cluster of dominating spin orientation at $T_{c}$, these are then occupied by the interacting Ising spins to simulate. Clusters for this thermally diluted Ising system have anomalous dimension $\eta_{\text {pure }} \approx 0.03$, which gives $a=2-\eta_{\text {pure }} \approx 1.97$. They obtain an exponent $\nu_{\mathrm{LRC}} \approx 1$, also in agreement with the WH expression. In both cases, LRC disorder is relevant for criticality. Nevertheless, this particular result from WH has proven recently not to be correct at more accurate approximations. Using two-loop expansions, Prudnikov et al. ${ }^{18}$ showed that the exponent $\nu_{\mathrm{LRC}}$ depends on both the internal dimension of the order parameter $m$ and the exponent $a$, not the case in WH's conjecture, independent of $m$. For both systems, the production of Prudnikovs' et al. yields $\nu_{\mathrm{LRC}} \approx 0.72 \neq 1$.

Experiments about the critical point of the liquid-vapor (LV) transition of ${ }^{4} \mathrm{He}$ and $\mathrm{N}_{2}$, confined in $95 \%$ porous aerogels, ${ }^{19,20}$ concern directly the problem we are addressing in this paper. Bulk ${ }^{4} \mathrm{He}$ near its LV critical point belongs to the 3DIS universality class, and aerogel-like disorder has proven to contain both LRC and SRC disordered structures. ${ }^{7,21}$ Wong et al. ${ }^{19,20}$ report, for the order parameter, exponents $\beta=0.28(5)$ and $0.35(5)$, respectively, consistent with that calculated for the pure 3DIS model by Guida and Zinn-Justin $^{22} \beta=0.326(3)$. However, in the same experiments, the specific heat curves present finite peaks at $T_{c}$, characteristic of a negative exponent $\alpha$, definitely different from the corresponding $\alpha_{\text {pure }} \approx 0.11$ for the pure 3DIS model. Actually, within error bars, results for $\beta$ are also consistent with the corresponding RDIS value, ${ }^{23} \beta=0.355(5)$. After these experimental results one may take noncorrelated SRC instead of LRC disorder within aerogels to be the relevant one for the critical behavior of the 3DIS model in aerogel pores. ${ }^{8}$

Renormalization group (RG) calculations for the 3DIS with weak amounts of disorder show that a new universality class appears, different from that of the pure 3DIS, ${ }^{12,13,24-28}$ and consistent with Harris criterion. Since Landau, ${ }^{29}$ using MC simulations, concluded that the exponents for the 3DIS model with random impurities differ from those of the pure system, different works stated that the exponents depend on the concentration of impurities, until 1990, when Heuer ${ }^{30,31}$ began to clarify that differences with RG calculations are due to the fact that exponents obtained from simulations were basically effective ones, and not the asymptotic ones.

Ballesteros et al. ${ }^{23}$ using a $p$-reweighting method in MC simulations, found the exponents for the RDIS universality class to be independent of the concentration of impurities $p$. This was confirmed for the random bond Ising model in $d$ $=3$ by Berche et al. ${ }^{32}$ looking at finite-size scaling for the critical temperature. All those calculations motivated further MC and RG studies about the crossover between the effective and really asymptotic critical behavior. ${ }^{15,28,32}$

Definitive evidence of a new universality class was obtained using neutron scattering in the antiferromagnets $\mathrm{Mn}_{1-x} \mathrm{Zn}_{x} \mathrm{~F}_{2}$ (Ref. 33) and $\mathrm{Fe}_{x} \mathrm{Zn}_{1-x} \mathrm{~F}_{2}$ (Ref. 34). For the first system, the exponents $\nu=0.70(2)$ and $\gamma=1.37(4)$ are obtained, while $\nu=0.69(2)$ and $\gamma=1.31(3)$ are the results for the second one. These results clearly differ from bulk exponents; see, for instance, those calculated by Guida and Zinn-Justin: ${ }^{22} \nu=0.6304(13)$ and $\gamma=1.239(5)$.

The critical behavior of magnetic systems confined in aerogel-like structures may be subject to competing LRC and SRC influences. The 3D $X Y$ model in the pores of DLCA aerogels, for instance, presents new exponents due only to the presence of gelling clusters, which are LRC, while the SRC components are irrelevant to the transition. ${ }^{7}$ However, for the Ising model under the same kind of confinement, two different effects may be present. Simulations under strictly LRC types of disorder, ${ }^{16,17}$ give exponents consistent with the result of Weinrib and Halperin. On the other hand, experimental results about the critical point of LV transitions in aerogels ${ }^{19,20}$ point to the relevance of the uncorrelated part of disorder.

\section{MODEL AND SIMULATION PROCEDURE}

The 3DIS model in the presence of impurities, on a simple cubic lattice with nearest-neighbor interaction, is described by the Hamiltonian

$$
\beta \mathcal{H}=-\frac{J}{k_{\mathrm{B}} T} \sum_{\langle i j\rangle} \epsilon_{i} \epsilon_{j} s_{i} s_{j},
$$

where $s= \pm 1$ are the spin variables, $k_{\mathrm{B}}$ is the Boltzmann constant, and $J$ is the coupling. In what follows, $k_{\mathrm{B}}=1$ and $T$ $=1$. The sets $\left\{\epsilon_{i}\right\}$ represent quenched variables chosen to be 0 if the site is an impurity and 1 if the site is occupied by an Ising spin. These sets of impurities are taken randomly (RDIS model) or from DLCA aerogels (AEIS model).

\section{A. Disorder generation and MC simulations}

At the beginning, sites are occupied by a uniform random distribution of $N$ particles, so their volume fraction is $c$ $=N / L^{3}$. To simulate the RDIS model, this initial distribution of disorder is held through the rest of the MC simulation.

Instead, for AEIS simulations to take place, aerogels are generated through the on-lattice DLCA algorithm ${ }^{9,10}$ with periodic boundary conditions ( $\mathrm{PBC}$ 's). Monomers and clusters diffuse randomly with diffusivity constants $D$ which depend on their mass $n$ through $D \sim n^{-1 / d_{f}}$. The fractal dimension $d_{f}$ has been taken equal to its value in three dimensions, ${ }^{35} d_{f}$ $\approx 1.8$. They stick irreversibly when they come in contact, and then the process follows up until a single cluster is obtained. This model is known to reproduce well the geometric features of real aerogels. ${ }^{36}$

At a given stage in this DLCA process, the first aggregate to reach opposite sides of the simulation box in any direction is called the gelling cluster (GC). It has been shown ${ }^{7,21}$ that 
the correlation function for this GC is algebraic up to a cutoff, which diverges as $L \rightarrow \infty$. In other words, these objects are fractal (LRC). Right after the GC is built, many other smaller clusters (islands) continue to diffuse and finally attach themselves to the GC at random sites. The resulting DLCA cluster (GC with islands) becomes homogeneous at a very small cutoff, in spite of the existence of a physically well-defined fractal structure, the GC. This cutoff increases as the concentration decreases, a feature already observed for real silica aerogels. ${ }^{6}$ At the volume fraction employed in the present work, $c=0.2$, the cutoff is so small (a few lattice constants) that DLCA clusters must be considered as nonfractals. Thus, the presence of islands, which represent the SRC subset within the whole DLCA cluster, actually hide the LRC behavior of gelling clusters. It is in this sense that aerogel-like structures must be considered as a mixture of LRC and SRC disorder distributions.

Disordered samples are generated by the procedures described above. A MC simulation is performed for each sample of interacting 3DIS spins, placed at empty sites left by impurities. Physical observables, denoted by $\mathcal{O}$, are calculated at each independent MC step, and then corresponding ensemble (thermal) averages $\langle\mathcal{O}\rangle$ are taken over the MC time series. The Wolff algorithm ${ }^{37}$ is used to update spins. In disordered systems, this algorithm tends to prevent some regions from being visited by growing Wolff clusters. If the concentration of impurities is small, this problem can be solved by adding some Metropolis updates along the simulation process. ${ }^{23}$ We chose this method and include some Metropolis sweeps to shake all spins, after a fixed number of Wolff steps. An independent step is taken after one correlation time $\tau$, which has been estimated from preliminary simulations. After enough steps for thermalization, a fixed number $\left(N_{T}=1000\right)$ of independent MC steps are performed to calculate thermal averages. Equivalent simulations take place for $N_{S}=2000$ different samples and, finally, averages over disorder are taken $O=[\langle\mathcal{O}\rangle]$ (denoted by square brackets). System sizes are $L=8,12,16,24,32,48,64,96$ for the RDIS model and $L=8,12,16,24,28,32,40,48,56$, $64,80,96$ for the AEIS model.

\section{B. Measured observables}

The magnetization (order parameter) is calculated by

$$
\mathcal{M}=\frac{1}{N} \sum_{i}^{L^{3}} \epsilon_{i} s_{i},
$$

where $N=c L^{3}$ is the total number of spins. Thermal averages $\langle\mathcal{M}\rangle$ are taken, and averages over disorder, $M(J)$ $=[\langle\mathcal{M}\rangle](J)$, are then calculated after the former have been extrapolated by reweighting. ${ }^{39}$ The procedure is described below in detail. In terms of the magnetization, we define the susceptibility as

$$
\chi=J L^{3}\left[\left\langle\mathcal{M}^{2}\right\rangle-\langle\mathcal{M}\rangle^{2}\right] .
$$

The energy is correspondingly defined by

$$
\mathcal{E}=-J \sum_{\langle i j\rangle} \epsilon_{i} \epsilon_{j} s_{i} s_{j}, \quad E=[\langle\mathcal{E}\rangle],
$$

and then the specific heat is obtained from fluctuations of the energy:

$$
c_{h}=L^{-3}\left[\left\langle\mathcal{E}^{2}\right\rangle-\langle\mathcal{E}\rangle^{2}\right] .
$$

Logarithmic derivatives of $n$th moments $M^{n}$ of the magnetization $(n=1,2,4)$, with respect to the coupling, are calculated through the energy-magnetization covariance:

$$
\left[\frac{\partial \ln \left\langle\mathcal{M}^{n}\right\rangle}{\partial J}\right]=-\left[\frac{\left\langle\mathcal{M}^{n} \mathcal{E}\right\rangle-\langle\mathcal{E}\rangle\left\langle\mathcal{M}^{n}\right\rangle}{\left\langle\mathcal{M}^{n}\right\rangle}\right] .
$$

\section{Simulation temperatures and reweighting}

Vásquez et al. ${ }^{7}$ found the phase diagram for the $3 \mathrm{D} X Y$ model in the pores of DLCA aerogels. They obtained $T_{c}(c) / T_{c}(0)=J_{c}(0) / J_{c}(c)$ as a function of the concentration of impurities $c$, being $J_{c}(c)$ the $3 \mathrm{D} X Y$ critical coupling at volume fraction $c$ of the aerogel. The shape of this phase diagram comes basically from the porous structure of disorder, especially at low concentrations. Using this information and the critical coupling $J_{c}(0)=0.2216595(26)$ for the pure 3DIS model, ${ }^{38}$ Vásquez $^{8}$ made a rough estimate for the critical coupling for the 3DIS model in the pores of DLCA aerogels at $c=0.2$. Making simulations at this rough estimate and using lattice sizes $L=10-80$ and finite-size scaling, the value $J_{c}(0.2)=0.25855(3)$ is obtained for the critical coupling. Although for those simulations the number of disorder realizations is low $\left(N_{\mathrm{S}}=30\right)$, they obtain critical exponents close enough to those reported for the RDIS model.

In the present work, all simulations were done at $c=0.2$, using simulation temperatures $\widetilde{J}_{c}=0.285745$ for the RDIS model (following Calabrese et al. ${ }^{15}$ ), and at the above estimate $\widetilde{J}_{c}=0.25855$ for the AEIS model. Physical quantities at $J \simeq \widetilde{J}_{c}$ are obtained by the reweighting method introduced by Ferrenberg and Swendsen. ${ }^{39}$ This procedure was used for each disorder realization at each system size $L$. Each thermodynamic quantity was then averaged over disorder for each $J$ within the extrapolation interval. Finally, maxima of $[\chi(J, L)],[c(J, L)]$ and $\left[\partial \ln \left\langle\mathcal{M}^{n}\right\rangle / \partial J(J, L)\right]$ were obtained from averaged curves, with their corresponding pseudocritical couplings $J_{c}^{*}(L)$.

\section{Disorder sampling and self-averaging}

This part is dedicated to determine a suitable number $N_{S}$ of disorder realizations to obtain critical exponents for the AEIS model at enough accuracy. A complete study of probability distributions for different thermodynamic quantities is performed and results compared with the corresponding ones for the RDIS model to decide $N_{S}$. As an example, in Fig. 1 we depict critical susceptibility points, obtained for corresponding disorder samples in the RDIS (left) and AEIS (right) cases (both at $c=0.2$ ). Points come from simulations at the couplings $\widetilde{J}_{c}$ estimated above, using the largest lattice sizes $(L=96)$. This distribution looks sharper in the AEIS 


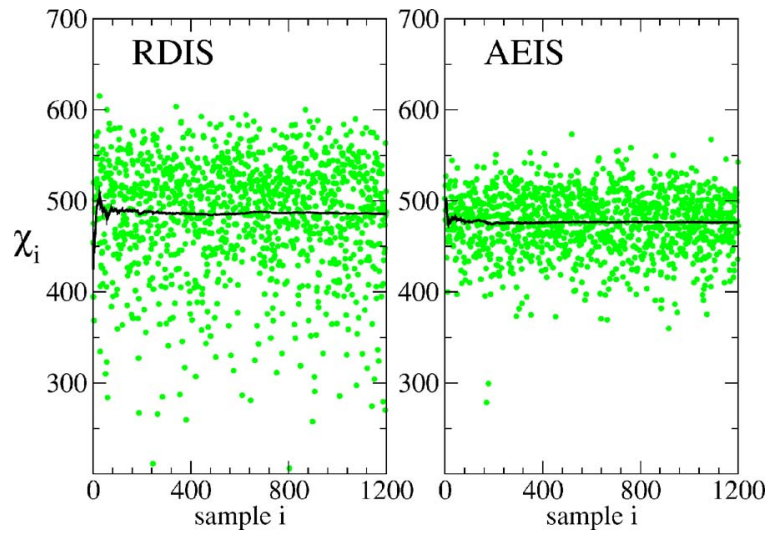

FIG. 1. (Color online) Distribution of the susceptibility for the RDIS (left) and AEIS (right) types of disorder, at a concentration of impurities $c=0.2$ and lattice size $L=96$. Simulations were performed at $\widetilde{J}_{c}=0.285745$ (Ref. 15) for the RDIS model and at $\widetilde{J}_{c}$ $=0.25855$ for the AEIS model. Running averages over the samples $\left[\chi_{i}\right]$ are shown by black thick solid lines.

case and notably more symmetric respect to the average than in the RDIS case. The top of Fig. 2 shows the probability distribution for the susceptibility in both cases.

As remarked, the distribution for the AEIS model (solid circles) is sharper and more symmetric than the distribution for the RDIS model (open squares). Note that, in terms of the
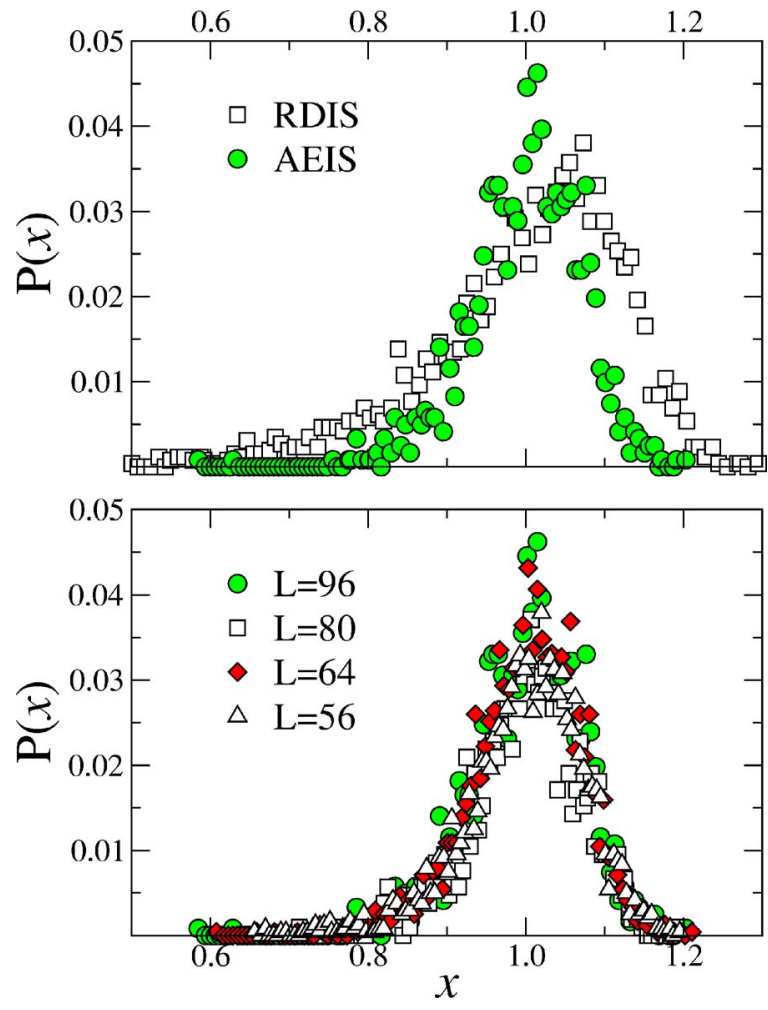

FIG. 2. (Color online) Probability distribution of the normalized susceptibility $x=\chi_{i} /[\chi]$. (Top) Distribution for the AEIS looks sharper and more symmetrical than that for the RDIS model (data taken from Fig. 1). (Bottom) Distributions at different lattice sizes for simulations of the AEIS model. The distribution width appears independent of the lattice size. normalized variable $x=\chi_{i} /[\chi]$, the maximum of the distribution for AEIS is closer to the average $x=1$ than for the RDIS model. The bottom of Fig. 2 shows the probability distribution of susceptibility for different lattice sizes. The independence of the width of distributions from $L$ is clear, typical for systems lacking self-averaging, which is the expected behavior of thermodynamic quantities for any disordered system at criticality. $^{41}$

Self-averaging can be quantitatively checked by the normalized squared width ${ }^{40} R_{A}$ :

$$
R_{A}(L)=\frac{\left[A^{2}(L)\right]-[A(L)]^{2}}{[A(L)]^{2}},
$$

$A$ being any given thermodynamic quantity. In this paper, $R_{A}(L)$ was estimated for the RDIS model to compare with previously reported values. We obtain, as $L \rightarrow \infty, R_{M}$ $\rightarrow 0.054$ for the magnetization and $R_{\chi} \rightarrow 0.016$ for the susceptibility, both in agreement with previous results. ${ }^{42}$ The ratio here obtained, $R_{M} / R_{\chi} \simeq 3.4$, disagrees with $\mathrm{RG}$ predictions: Aharony and Harris ${ }^{43}$ obtained, using $\epsilon=4-d$ expansions, that the leading term is $R_{M} / R_{\chi}=1 / 4$. The discrepancy may come from higher-order terms in the expansion, and not from the definition of the susceptibility as was suggested by Berche et $a .^{32}$ Note also that, in the present work, the definition for the susceptibility, $\chi=J L^{3}\left[\left\langle\mathcal{M}^{2}\right\rangle-\langle\mathcal{M}\rangle^{2}\right]$, differs from that used by Wiseman and Domany, ${ }^{42} \chi=J L^{3}\left[\left\langle\mathcal{M}^{2}\right\rangle\right]$.

Results for $R_{A}(L)$, plotted versus $L^{-1}$, in the AEIS case, are shown in Fig. 3, $A$ being the order parameter $M, \chi$ the susceptibility, $c_{h}$ the specific heat, and $E$ the energy, respectively. As observed for $M, \chi$, and $c_{h}$ (top), $R_{A}$ tends to nonzero values as $L \rightarrow \infty$, though asymptotic limits for $R_{A}$ are smaller for the AEIS than for the RDIS model, as expected $\left(R_{M} \rightarrow 0.020\right.$ and $R_{\chi} \rightarrow 0.0028$, as $\left.L \rightarrow \infty\right)$. The power-law behavior $R_{E} \sim L^{-x}$ has been depicted for the energy (bottom), the fitting exponent being $x \simeq 2.58$. Thus, the energy is weakly self-averaged $^{41}(x<d)$. The same type of behavior is obtained, in this work, for the energy in the RDIS case, in agreement with previously reported results. ${ }^{41}$ According to these analyses, the number $N_{S}$ of disorder realizations, suitable to estimate critical exponents, is larger for the RDIS than for the AEIS model. In the next section, we report some values for the effective critical exponents for both models. Our results for the RDIS model agree well with previously published results; ${ }^{42}$ thus, the same number of realizations for the AEIS model will be enough to estimate critical exponents, as the values of the normalized squared widths $R_{A}$ are substantially lower than those obtained for the RDIS model.

\section{E. Critical couplings}

We use two methods to estimate the critical coupling out of our present simulations. The Binder magnetization fourth cumulant

$$
U_{4}=1-\frac{\langle M\rangle^{4}}{3\left\langle M^{2}\right\rangle^{2}}
$$

is universal-i.e., independent of the system $\operatorname{size}^{45}$ at the critical point. Thus, the critical coupling $J_{c}$ can be obtained 


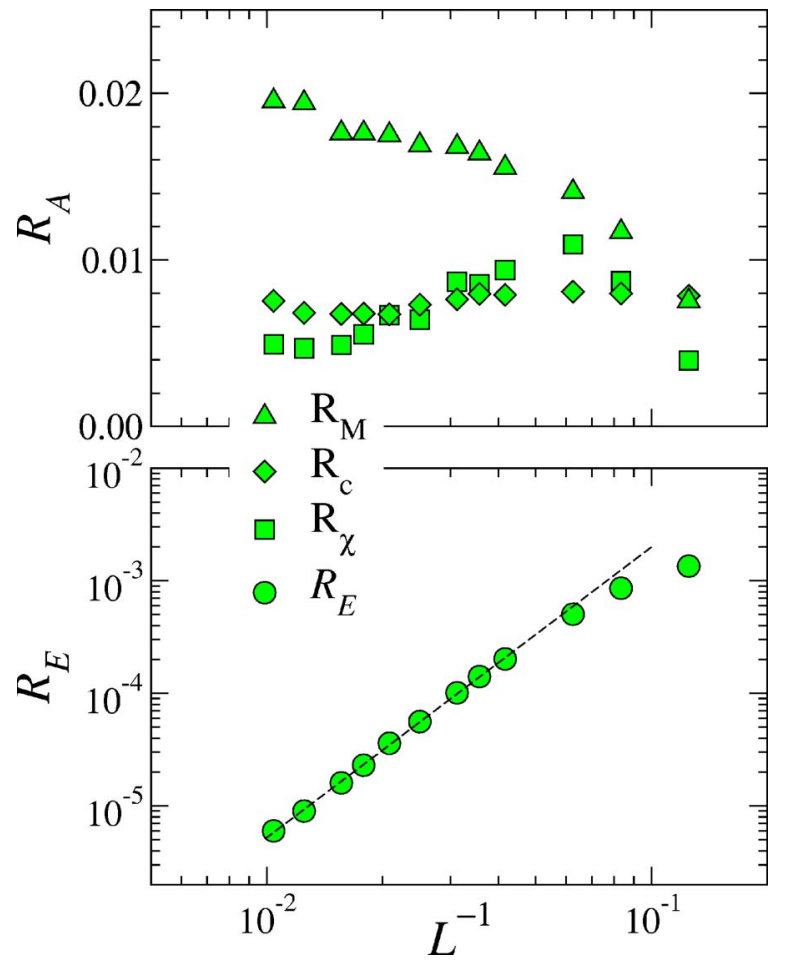

FIG. 3. (Color online) Normalized squared widths $R_{A}$ versus inverse system size $L^{-1}$ for the AEIS model at criticality. (Top) Asymptotic nonzero values for $R_{A}$ as $L \rightarrow \infty$, for the magnetization $(M)$, the susceptibility $(\chi)$, and the specific heat $\left(c_{h}\right)$, evidence the lack of self-averaging in these quantities. (Bottom) The power-law behavior $R_{E} \sim L^{-x}$, with a fitting exponent $x \simeq 2.58<d$, indicates that the energy $(E)$ is weakly self-averaged.

with high accuracy at the point where the $U_{4}-J$ plots coincide for all system sizes $L$. Figure 4 shows these plots for the largest system sizes, for the RDIS (top) and AEIS (bottom) models. In both cases, the coupling used in the simulations has been marked by a vertical dashed line. For the RDIS case, taking the curves for the two largest sizes $(L=64,96)$, we obtain an estimate (circle) $J_{c}^{R D I S}=0.2857471$ (11) for the critical coupling, which agrees well with that of Calabrese et al. ${ }^{15} J_{c}^{R D I S}=0.2857447(24)$. In the AEIS case, we depict the same plots for lattice sizes $L=56-96$. The intersection for $L=80,96$ (circle) gives the critical coupling $J_{c}^{\text {AEIS }}$ $=0.258575(10)$, close to the value used in the simulations, $J_{c}^{A E I S}=0.25855\left(\Delta J_{c} / J_{c} \approx 10^{-4}\right)$.

On the other hand, following the finite-size scaling (FSS) theory ${ }^{45}$ deviations of pseudocritical couplings $J_{c}^{*}(L)$ from the critical coupling $J_{c}$ scale as

$$
J_{c}^{*}(L)-J_{c} \sim L^{-1 / \nu}
$$

where $J_{c}^{*}(L)$ is defined as the positions of maxima for a given critical quantity, being $J_{c} \equiv J_{c}^{*}(L \rightarrow \infty)$. For instance, values $J_{c}^{*}(L)$ for the susceptibility (7) and logarithmic derivatives (8), obtained from reweighted curves, are depicted in Fig. 5 as functions of $L^{-1 / \nu}$, where the rough value $1 / \nu \approx 1.4$ has been estimated through nonlinear fits of points corresponding
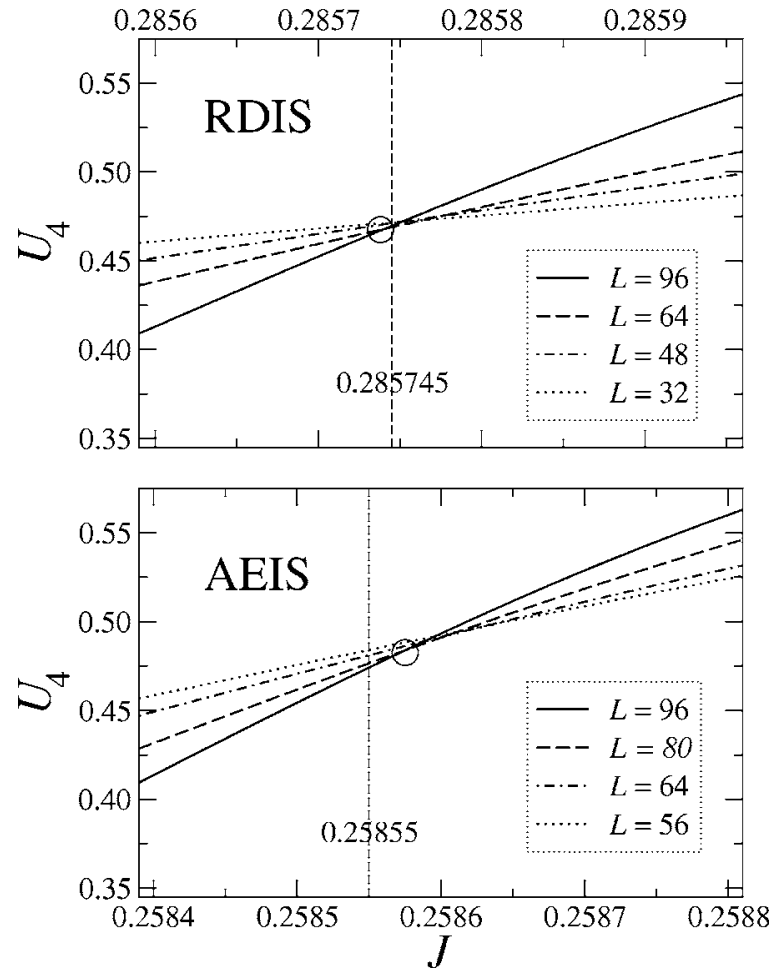

FIG. 4. Binder magnetization fourth cumulant as a function of $J$ for the RDIS (top) and the AEIS (bottom) models, using the largest $L$. Dashed vertical lines indicate couplings $\widetilde{J}_{c}$ used in simulations; open circles are intersections between curves at the two largest sizes. In the AEIS case, $J_{c}$ from this intersection and the previous estimate $\widetilde{J_{c}}$ differ by $\Delta J_{c} / J_{c} \approx 10^{-4}$.

to $L=56-96$. As expected, the linear behavior (11) is observed and the lines cross the $L \rightarrow \infty$ axis at an average point $J_{c}=0.258570(13)$, quite close to the value estimated above, using the intersection of Binder fourth cumulants.

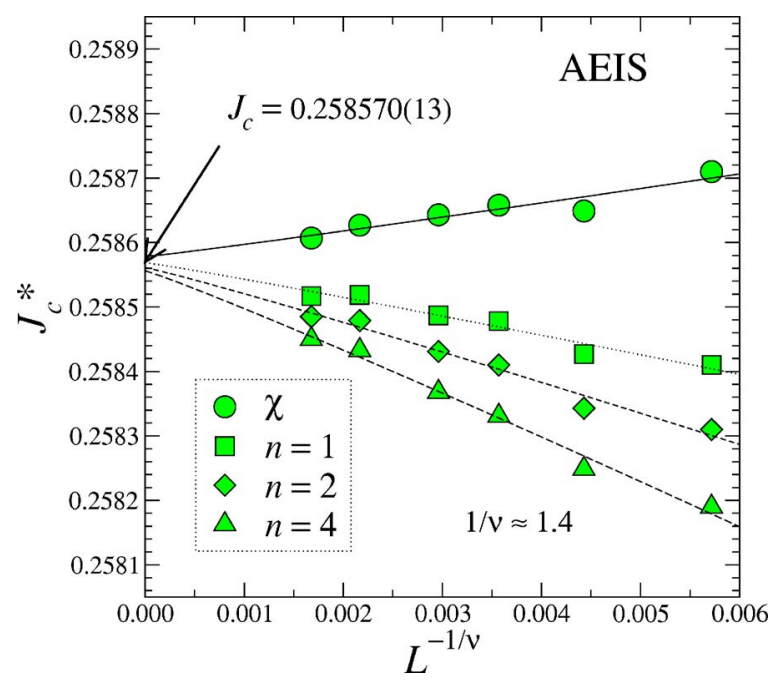

FIG. 5. (Color online) Position of the maxima $J_{c}^{*}$ for the susceptibility and logarithmic derivatives of $M^{n}(n=1,2,4)$, plotted versus $L^{-1 / \nu}$ using $1 / \nu=1.4$. Linear fits average at $J=0.258570(13)$ in the thermodynamic limit $(L \rightarrow \infty)$. 


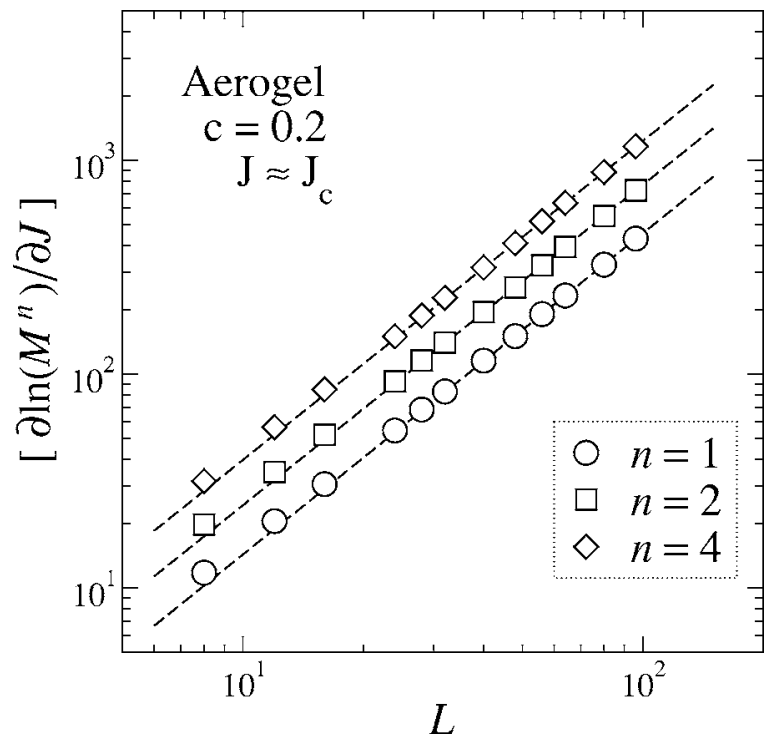

FIG. 6. Logarithmic derivatives $\left[\partial \ln \left\langle\mathcal{M}^{n}\right\rangle / \partial J\right](n=1,2,4)$ versus $L$. Points are averages over disorder taken from our simulations at the estimate $\widetilde{J}_{c}=0.25855$ for the AEIS model. Power-law fits (dashed lines) out of the four largest lattice sizes $L=56-96$ give a FSS exponent $1 / \nu=1.501 \pm 0.007$.

\section{EFFECTIVE EXPONENTS}

\section{A. Correlation length exponent}

Finite-size scaling ${ }^{44}$ has been used to estimate effective critical exponents for the RDIS and AEIS cases. This method allows us estimate the critical exponents $\beta / \nu, \gamma / \nu$, and $\alpha / \nu$ and the correlation length inverse exponent $1 / \nu$. The latter has already been roughly estimated above, using the scaling law for the position of the maxima of logarithmic derivatives of the magnetization moments, ${ }^{38}\left\langle\mathcal{M}^{n}\right\rangle(n=1,2,4)$, and the susceptibility. More accurate estimations are made directly taking averages over disorder on quantities obtained at $J_{c}$ $=0.25855$, used in our extensive simulations, which is close to the previously estimated $J_{c}$ :

$$
\left[\frac{\partial \ln \left\langle\mathcal{M}^{n}\right\rangle}{\partial J}\right]_{J=J_{c}} \sim L^{1 / \nu} .
$$

We look first to this exponent in order to determine the effect of disorder on the critical behavior of the 3DIS model. Previous works ${ }^{32}$ report that the exponents $\beta / \nu$ and $\gamma / \nu$ for the RDIS model are almost the same as those for the pure 3DIS model, and as shown later, this is the case for the AEIS model.

Logarithmic derivatives of moments $n=1,2,4$ of the magnetization are plotted, versus system size $L$, in Fig. 6 for the AEIS case. Points were obtained from averages over disorder at the simulation coupling $\widetilde{J_{c}}=0.25855$ which is quite close to $J_{c}$, as estimated above. Dashed lines are power-law fits to Eq. (12) using the four largest system sizes ( $L$ $=56-96)$ and give a FSS exponent $1 / \nu=1.501 \pm 0.007$ for the AEIS model. We recall that exponents determined by this method are effective exponents and only their asymptotic

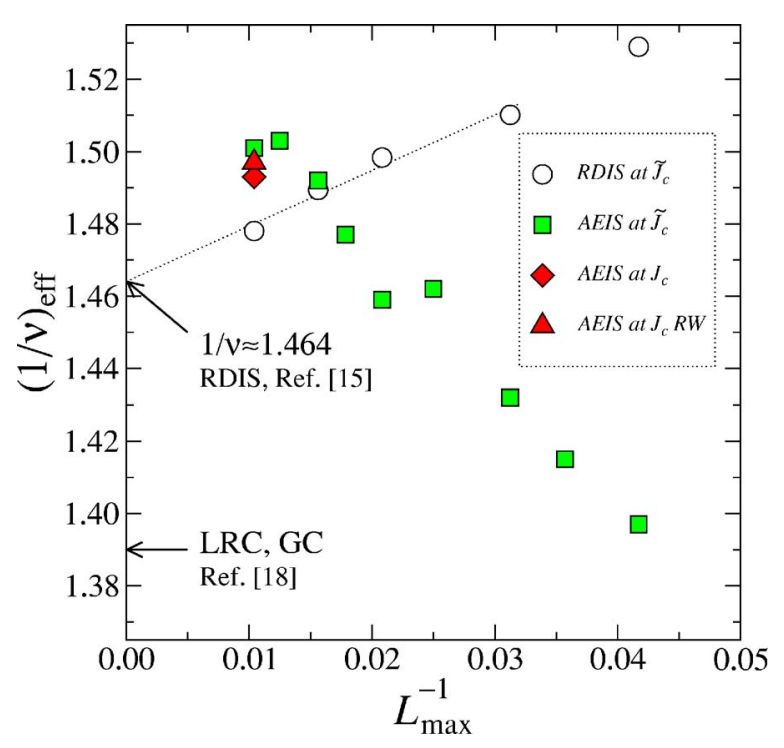

FIG. 7. (Color online) Effective correlation length inverse exponent $(1 / \nu)_{\text {eff }}$ versus $1 / L_{\max }$, obtained by the FSS power-law fit (12) of four consecutive points ending at $L_{\max }$. Values for the RDIS (open circles), extrapolated to $L_{\max } \rightarrow \infty$ (dotted line), approach the result by Calabrese et al. (Ref. 15). In the AEIS case (solid squares), points clearly depart from the LRC fixed point through a region close but above the SRC fixed point. Averages over disorder on less extensive simulations of the AEIS at the critical coupling $J_{c}$ $=0.258570$ give $(1 / \nu)_{\mathrm{eff}} \simeq 1.493$ (diamond). The value $(1 / \nu)_{\mathrm{eff}}$ $\simeq 1.497$ (triangle) is obtained from averaged reweighted curves at $J_{c}=0.258570$.

behavior would give a hint as to what the universal critical exponent tends to. This study is addressed in the next paragraphs.

Effective exponents $(1 / \nu)_{\text {eff }}$ are depicted in Fig. 7 as calculated from FSS of logarithmic derivatives in both cases, RDIS and AEIS. As in Fig. 6, values were obtained from averages over disorder at the simulated couplings $\widetilde{J_{c}} \approx J_{c}$, being $\widetilde{J}_{c}=0.285745$ for the former and $\widetilde{J}_{c}=0.25855$ for the latter, as stated above. Each value $(1 / \nu)_{\text {eff }}$ is then obtained from power-law fits to the FSS expression (12), taking four consecutive points whose maximum size is $L=L_{\max }$. Results for the RDIS (open circles) yield $(1 / \nu)_{\mathrm{eff}}=1.478(5)$ at $L_{\max }$ =96. A rough estimate of the asymptote $1 / \nu$ is obtained by extrapolating these points to the $L_{\max }^{-1} \rightarrow 0$ axis, as seen in Fig. 7 (dotted line). The extrapolation yields $1 / \nu \approx 1.464$, well in agreement with previously reported results for the RDIS model. ${ }^{15}$ Results for the AEIS model (solid squares) give $(1 / \nu)_{\mathrm{eff}}=1.501(7)$ at $L_{\max }=96$. Effective exponents in this case clearly depart from values corresponding to the LRC fixed point, ${ }^{18}$ through a region close but above the SRC fixed point at $L_{\max } \approx 48$. However, at larger lattice sizes, greater values suggest that a another fixed point may rule the critical behavior at the thermodynamic limit.

For the stable uncorrelated (SRC) disorder fixed point, the theory ${ }^{1}$ predicts that the exponent $1 / \nu$ should be smaller than $3 / 2$. Additionally, the $\mathrm{WH}$ condition $^{2}$ (2) is well satisfied for this AEIS model, where $\nu$ is the pure 3DIS exponent and $a$ $=2\left(d-d_{f}\right)$ comes from LRC of GC's within DLCA aerogels. ${ }^{7}$ 
In effect, the fractal dimension for the GC's within aerogels at $c=0.2$ is $d_{f} \approx 2.2$, as reported elsewhere. ${ }^{7,8}$ This condition, together with theoretical predictions reported by Prudnikov et al.,$^{18}$ would give $1 / \nu \approx 1.4$ for the 3DIS model with LRC defects, at the corresponding $a \approx 1.6$. From Fig. 7 it is clear that $(1 / \nu)_{\text {eff }}$ is far above this value. Thus, it is not the LRC subset of disorder (the GC's) which rules the critical behavior of the AEIS model, in the way it certainly does for 3D $X Y$ universality class in aerogels. ${ }^{7}$

The exponent $(1 / \nu)_{\text {eff }}$ at $L_{\max }=96$ was also obtained taking averages of logarithmic derivatives from average reweighted curves, at the critical coupling estimated above (Sec. III E), $J_{c}=0.258570$. For $L=56-96$ we obtain $(1 / \nu)_{\text {eff }} \approx 1.497$ (triangle, Fig. 7). In addition, less extensive additional realizations of the AEIS model also for $L$ $=56-96(600$ for each size $)$ were made at this more accurate value $J_{c}=0.258570$ and averages over disorder were taken directly from simulations. The power-law fit for these points gives an estimate $(1 / \nu)_{\text {eff }} \approx 1.493$ (diamond). Although these effective exponents are lower than $3 / 2$, there exists yet not enough evidence in this work that the RDIS fixed point would be reached at $L_{\max } \rightarrow \infty$.

\section{B. Specific heat and energy exponents}

To check our results about the correlation length exponent for the AEIS, we study the FSS of the specific heat and the energy, at the simulation coupling $\widetilde{J}_{c}$.

The top of Fig. 8 shows the specific heat $c_{h}$ plotted versus $L$ using a linear-log scale. The dashed line is a logarithmic fit using the four largest lattice sizes 56-96. This result suggests that the singularity of the specific heat could be logarithmic, consistent with our result $1 / \nu \approx 1.5$ for $L_{\max }=96$. After the Josephson hyperscaling relation $(\alpha=2-d \nu)$ a specific heat exponent $\alpha \approx 0$ would be expected. The bottom of Fig. 8 shows the linear dependence of the energy $E$ on $L^{(\alpha-1) / \nu}$, taking $\nu=2 / 3$ and $\alpha=0$, which confirms the results stated above. We made an additional analysis of the specific heat data, and the energy as well, using the scaling of both quantities in the case $\alpha / \nu<0$. This method was performed by Schultka and Manousakis in determining the (negative, very small) exponent $\alpha / \nu$ for the pure 3D $X Y$ model. ${ }^{46}$ In the case $\alpha / \nu<0$, the specific heat scales as $c_{h}=c_{\infty}+c_{1} L^{\alpha / \nu}$, while the energy scales as $E=E_{\infty}+E_{1} L^{(\alpha-1) / \nu}$. Nonlinear fits to these expressions, using the six largest lattice sizes 40-96, give the self-consistent results $\alpha / \nu \approx-0.022$ and $(\alpha-1) / \nu \approx-1.512$, in agreement with a correlation length exponent $1 / \nu \approx 1.49$. This result agrees with the tendency shown by the effective values in Fig. 7. Experiments on the critical point of the LV transition of ${ }^{4} \mathrm{He}$ in aerogels ${ }^{19}$ report a cusplike peak in the specific heat, but the authors do not report an estimate for the exponent $\alpha$. Our results point for the largest lattice sizes to a logarithmic singularity, which may also be consistent with these experimental results.

\section{Magnetic exponents}

According to the FSS theory, the magnetization and the susceptibility scale as $M \sim L^{-\beta / \nu}$ and $\chi \sim L^{\gamma / \nu}$, respectively. In
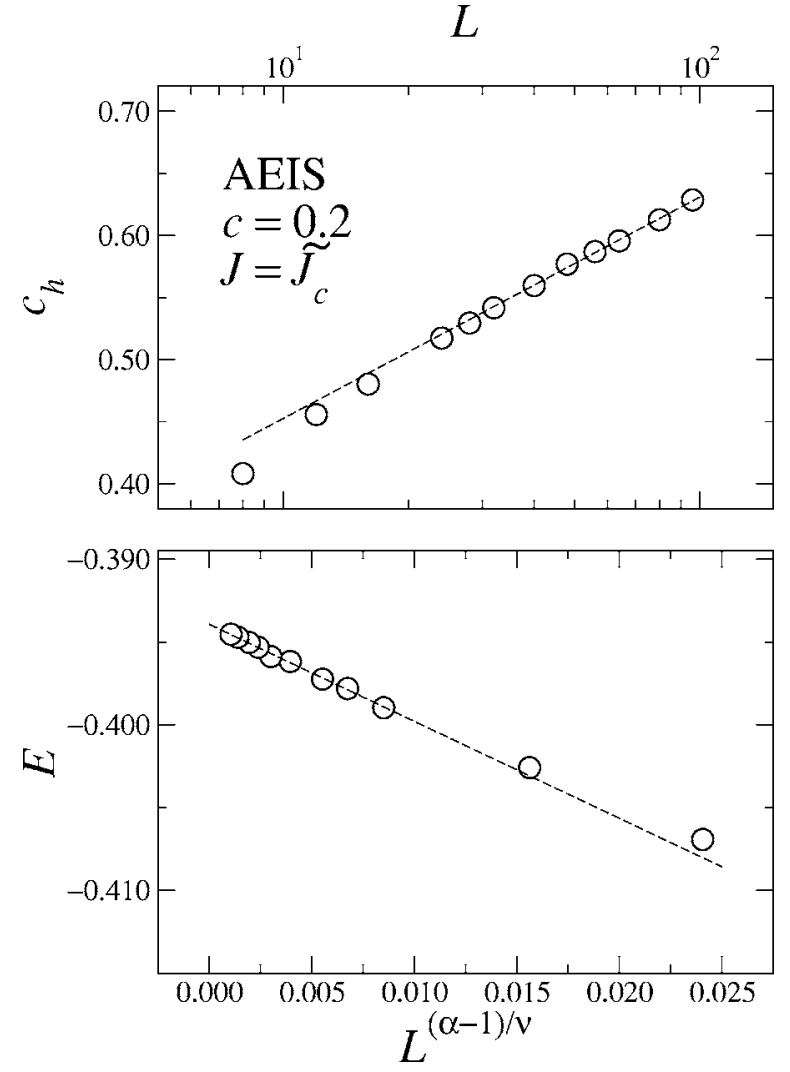

FIG. 8. (Top) Specific heat $c_{h}$ versus $L$ for the AEIS in a linear$\log$ scale. A logarithmic singularity $(\alpha=0)$ for the specific heat at $\widetilde{J}_{c}$ follows from the fit (dashed line). This result reinforces the estimated $1 / \nu \approx 1.5$ (Fig. 6). (Bottom) Energy plotted against $L^{(\alpha-1) / \nu}$, taking $\alpha=0$ and $1 / \nu=3 / 2$, and the corresponding linear fit is shown (dashed line).

Fig. 9, we plot the order parameter $M$ (left) and the susceptibility (right) as a function of $L$ for the AEIS model. Magnetization data (circles) have been fit to the preceding powerlaw FSS expression (solid line), giving $\beta / \nu=0.523(3)$. The average sizes of Wolff clusters divided by $L^{3},\left[\left\langle n_{\mathrm{W}}\right\rangle\right]$ (squares), scale with the same exponent as the squared magnetization. ${ }^{47}$ This is confirmed by the power-law fit (dashed line) which yields $2 \beta / \nu=1.019(6)$. These results give an average estimate $\overline{2 \beta / \nu}=1.032(6)$. Together with $1 / \nu \approx 1.5$ this gives $\beta=0.34(4)$, close to the pure 3DIS exponent and to the RDIS exponent. On the right side of Fig. 9, points for the susceptibility obtained from simulations near the critical point fit to the FSS power-law expression with the exponent $\gamma / \nu=2.044(4)$. All fits have been made for $L$ $=56-96$.

The results for magnetic effective FSS exponents, obtained by fitting four consecutive points from Fig. 9, ending at $L_{\max }$, are depicted in Fig. 10. Figure 10(a) shows the effective exponent for the susceptibility, $(\gamma / \nu)_{\text {eff }}$, plotted versus $L_{\max }^{-1}$. This exponent tends to increase for $L_{\max }<48$, but beyond this size the tendency is to stabilize at a value close to that of percolation, $\gamma / \nu=2-\eta \approx 2.05$. For larger $L_{\max }$, it turns to approach a value lower than 2.00 (positive $\eta$ ). The asymptotic extrapolated value seems to be the pure 3DIS exponent $\gamma / \nu=1.966$ (3) (Ref. 22) or the RDIS exponent ${ }^{23}$ 


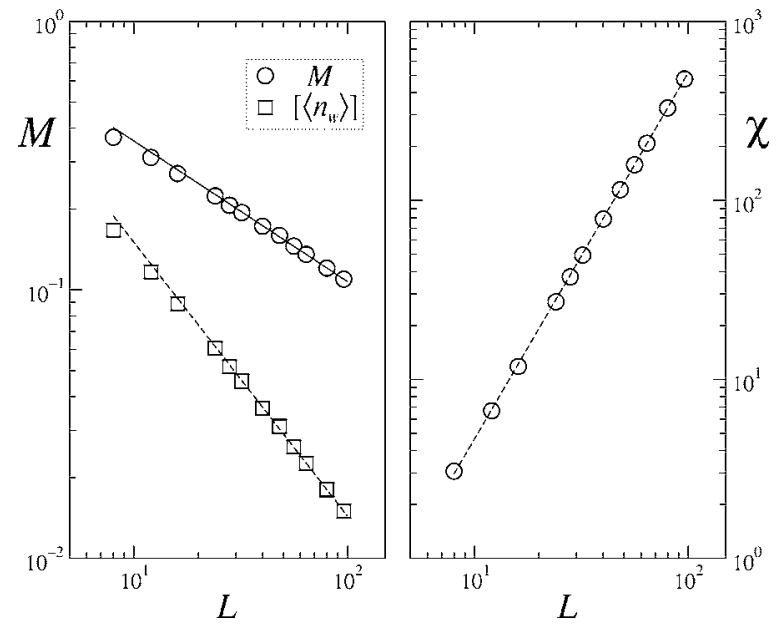

FIG. 9. Order parameter (left) and susceptibility (right), at the simulated critical point $\widetilde{J}_{c}$, versus $L$ for the AEIS model. The magnetization $M$ (circles) scales as $L^{-\beta / \nu}$, and the averaged Wolff cluster size $\left[\left\langle n_{\underline{\mathrm{W}}}\right\rangle\right]$ scales as ${ }^{47} L^{-2 \beta / \nu}$ (squares), giving an average estimate of $2 \beta / \nu=1.032(1)$. The FSS power-law fit for the susceptibility gives $\gamma / \nu=2.044(4)$. All power-law fits have been made for $L=56-96$.

$\gamma / \nu=1.963(5)$. Berche and collaborators ${ }^{32}$ estimated effective values $\gamma / \nu>2$ for smaller concentrations in the RDIS case. Using the result for $L_{\max }=96, \gamma / \nu=2.044(4)$, and our estimate $\nu=2 / 3$, the exponent $\gamma$ found in the present work is 1.363(9), slightly above the value $\gamma=1.344(9)$ found in our MC simulations for the RDIS model, and $\gamma=1.342$ obtained by Calabrese et al. ${ }^{15}$ for the same system. The tendency for larger $L$ is to be closer to RDIS results.

We must remind the reader at this point that the theoretically most accurate results, by Prudnikov et al. ${ }^{18}$ predict a magnetic exponent $\eta<0$ for the 3DIS model in LRC disordered structures with an algebraic decay similar to that of the gelling clusters within DLCA aerogels at $c=0.2{ }^{8}$

In Figs. 10(b) and 10(c), squares represent effective exponents $(\beta / \nu)_{\text {eff }}$ obtained from $\left[\left\langle n_{\mathrm{W}}\right\rangle\right]$ and circles those obtained directly from $M$. There is a strong variation of these results with $L_{\max }$. As stated above, the exponent obtained averaging both results using $L_{\max }=96$ is $\overline{\beta / \nu}=0.516(6)$, close to that of the pure 3DIS model, $\beta / \nu=0.517(3) .{ }^{22}$ In addition, our results agree well with those reported for the RDIS case by Ballesteros et al. ${ }^{23} \beta / \nu=0.519$ (3). In this work, we obtain $\beta / \nu=0.516(5)$ for the RDIS model. The last effective value $(\beta / \nu)_{\text {eff }}\left(L_{\max }=96\right)$, together with $1 / \nu \approx 1.5$, gives an exponent $\beta=0.343(9)$ for the order parameter. This result agrees well with experiments about the critical point of the LV transition of $\mathrm{N}_{2}$ in $95 \%$ porous aerogels $(c=0.05)$, reported by Wong et al. ${ }^{20}$ which yield $\beta=0.35(5)$.

Care must be taken with this agreement because our results were obtained using DLCA at concentrations $c=0.2$ and these structures are quite different from those at $c=0.05$. For the latter, most of impurities belong to the LRC gelling clusters, giving the DLCA aerogels a less random overall structure.

As stated above, a possible explanation for these magnetic exponents is the influence of the LRC disorder fixed point.

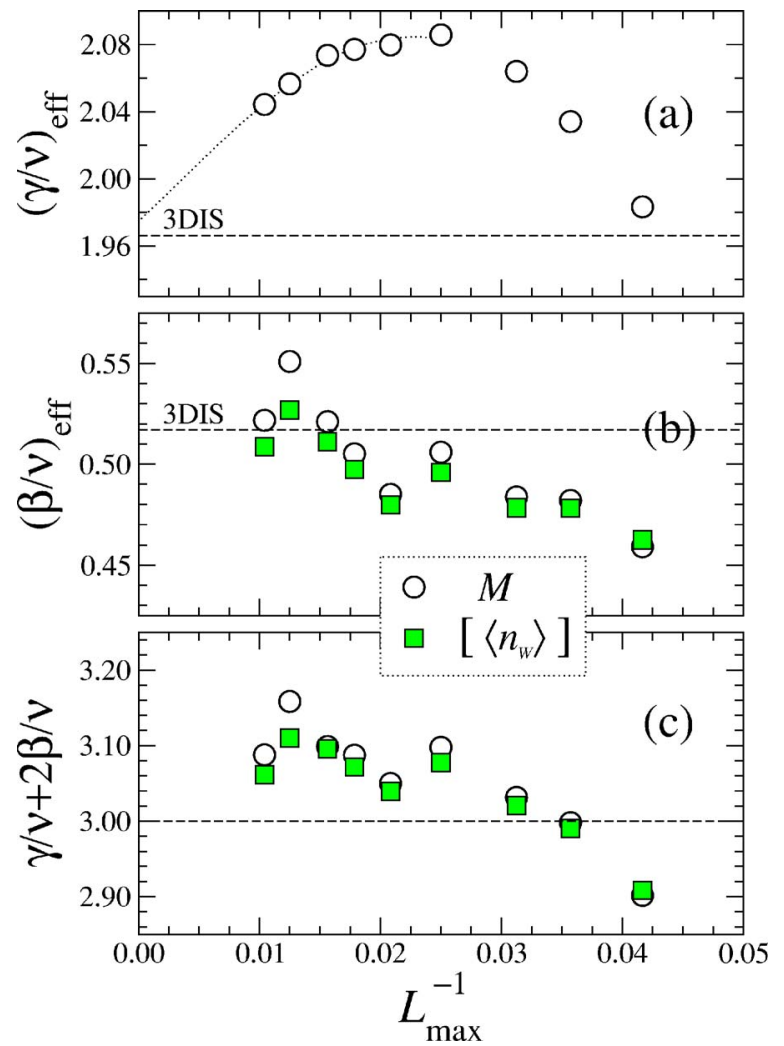

FIG. 10. (Color online) (a) Effective exponents for the susceptibility give $(\gamma / \nu)_{\text {eff }}=2-\eta_{\text {eff }}>2$. Extrapolation using $L_{\text {max }} \geq 48$ suggest that $\gamma / \nu<2$ in the thermodynamic limit. (b) Effective exponents $(\beta / \nu)_{\text {eff }}$ for the magnetization (circles) and for the average mass of Wolff clusters (squares). At $L_{\max }=96$ both approach the pure 3DIS theoretical estimate (Ref. 22) $(\beta / \nu)_{\text {eff }} \approx 0.517$. (c) The hyperscaling relation $\gamma / \nu+2 \beta / \nu=d=3$, not satisfied for effective exponents, tends to hold for larger lattice sizes.

The fractal dimension of the aerogel gelling cluster is $d_{f}$ $\approx 2.2$, giving an exponent $a \approx 1.6$ associated with this structure. ${ }^{7}$ Following Table IV from Prudnikov et al. ${ }^{18}$ a value $\gamma / \nu>2.0205(\eta<-0.0205)$ is expected. The effective value found in this work is close to this prediction, but it follows from Fig. 10(b) that a tendency exists to approach a value closer to the corresponding RDIS fixed point. Finally, using effective values the hyperscaling relation $\gamma / \nu+2 \beta / \nu$ $=3$ seems not to hold, as seen in Fig. 10(c). Violation of this hyperscaling relation suggests that our results do not yet reach asymptotic values. Extensive simulations still need to be performed at the more accurate value $J_{c}=0.258570$.

\section{CONCLUDING REMARKS}

Extensive Monte Carlo simulations of the 3D Ising model with impurities have been reported in this paper. Using finite-size scaling, critical couplings and exponents have been estimated for the 3DIS model, in the presence of randomly distributed impurities (RDIS model) and confined in aerogel-like structures (AEIS model). For the latter we have collocated Ising spins in the pores of simulated DLCA aerogels at $c=0.2$. At this concentration, these objects are known 
to be nonfractal. However, the presence of hidden LRC could affect criticality, as predicted by the theory. ${ }^{18}$ It has been concluded elsewhere ${ }^{7}$ that these LRC structures, the gelling clusters, modify the critical behavior of the 3D $X Y$ model when confined in the same kind of aerogel-like structures. In the 3DIS case, however, our results for the thermal exponents $1 / \nu \lesssim 1.5$ and $\alpha / \nu \lesssim 0$ rest far above those for the LRC fixed point predicted by the theory. ${ }^{18}$ Complementary simulations at a more accurate value of the critical coupling, $J_{c}$ $=0.258570(13)$, give an exponent $1 / \nu \approx 1.49$. Although similar thermal exponents have been reported by Pakhnin and Sokolov $^{48}$ for the RDIS universality class, the asymptotic critical regime could have not been reached in our simulations and more extensive simulations are yet to be performed at this more accurate $J_{c}$ value.

Effective critical exponents observed here for the AEIS change from a fixed point (LRC) at box sizes $L \leq 48$ to another (SRC) at box sizes $L>48$ (Figs. 7 and 10), probably indicating an oscillating approach to the stable fixed point. Theoretical predictions based on the Weinrib-Halperin model, ${ }^{2,18}$ able to explain changes of the critical behavior of the 3D $X Y$ model in the pores of DLCA aerogels, ${ }^{7}$ may also explain the influence of this type of disorder on the 3DIS model. In this case, two competing effects are present: the random SRC subset of the disorder (defined in Sec. III A), which already affects the critical behavior of the 3DIS model, and the LRC subset which, after the extended criterion (2), may be relevant as well. This is certainly not the case for the 3D $X Y$ model, where the Harris criterion prevents the SRC subset of impurities (islands) from being relevant: only the weak LRC distribution of impurities (GC) is relevant ${ }^{7}$ for the $3 \mathrm{D} X Y$ model. For the 3DIS model, theory predicts that both LRC and SRC subsets may be relevant. Which one finally dominates the critical behavior?

The results presented in this paper suggest that, in the AEIS case, the critical behavior is ruled by the SRC fixed point. A plausible explanation of these dominating SRC effects is provided by theoretical works: ${ }^{2,18}$ for the 3DIS model, RG flows converge to a more stable SRC fixed point, because at $m=1$ the LRC fixed point is less stable (marginal). However, it has been mentioned before, without a proof, ${ }^{2}$ that the amplitudes of disorder may in some cases affect criticality. Added in proof, we have to mention that in preliminary simulations of the 3DIS model in the presence of mixed kinds (LRC and SRC) of disorder, ${ }^{49}$ evidence of a continuous flow from the LRC fixed point to the SRC one has been observed when relative strengths are tuned from a pure LRC distribution of defects to a 1:1 proportion. In the AEIS case, we have analyzed the relative amplitudes (strengths) of the LRC and SRC subsets of disorder, for $L$ $=128$, to determine that up to $97 \%$ of defects are due to islands (SRC), while only 3\% are due to the GC's (LRC).

To conclude, it has to be stated that the influence of aerogel-like distributions of impurities on the critical behavior of the 3DIS model is yet far from being completely understood. The problem is similar to that of phase transitions in Ising systems with noninteger dimension or in fractal structures. It becomes clear that the fractal dimension, related to the exponent $a$ of subjacent long-range correlations, may not be the only parameter to determine the universality class of the impure system. ${ }^{50}$

\section{ACKNOWLEDGMENTS}

The authors thank CNRS and FONACIT (Grant No. PI2004000007) for their support. Discussions with N. OliviTran, B. Berche, Yu. Holovatch, and M. Marqués are kindly acknowledged. Invaluable discussions with A. Hasmy and R. Jullien have improved our understanding of aerogel structure. C.V. kindly expresses gratitude to all the personnel of the LCVN at Montpellier, France.
${ }^{1}$ A. B. Harris, J. Phys. C 7, 1671 (1974).

${ }^{2}$ A. Weinrib and B. I. Halperin, Phys. Rev. B 27, 413 (1983).

${ }^{3}$ M. H. W. Chan, K. I. Blum, S. Q. Murphy, G. K. S. Wong, and J. D. Reppy, Phys. Rev. Lett. 61, 1950 (1988).

${ }^{4}$ J. Yoon, D. Sergatskov, J. Ma, N. Mulders, and M. H. W. Chan, Phys. Rev. Lett. 80, 1461 (1998).

${ }^{5}$ J. Yoon and M. H. W. Chan, Phys. Rev. Lett. 78, 4801 (1997).

${ }^{6}$ R. Vacher, T. Woignier, J. Pelous, and E. Courtens, Phys. Rev. B 37, 6500 (1988).

${ }^{7}$ C. Vásquez, R. Paredes V., A. Hasmy, and R. Jullien, Phys. Rev. Lett. 90, 170602 (2003); C. Vásquez, R. Paredes V., A. Hasmy, and N. Olivi-Tran (unpublished).

${ }^{8} \mathrm{C}$. Vásquez, Ph.D. thesis, Instituto Venezolano de Investigaciones Científicas, Caracas, 2003.

${ }^{9}$ P. Meakin, Phys. Rev. Lett. 51, 1119 (1983).

${ }^{10}$ M. Kolb, R. Botet, and R. Jullien, Phys. Rev. Lett. 51, 1123 (1983).

${ }^{11}$ G. Grinstein and A. Luther, Phys. Rev. B 13, 1329 (1976).

${ }^{12}$ Yu. Holovatch and T. Yavors'kii, J. Stat. Phys. 92, 785 (1998).

${ }^{13}$ R. Folk, Yu. Holovatch, and T. Yavors'kii, Phys. Rev. B 61,
15114 (2000).

${ }^{14}$ A. Pelissetto and E. Vicari, Phys. Rev. B 62, 6393 (2000).

${ }^{15}$ P. Calabrese, V. Martín-Mayor, A. Pelissetto, and E. Vicari, Phys. Rev. E 68, 036136 (2003).

${ }^{16}$ H. G. Ballesteros and G. Parisi, Phys. Rev. B 60, 12912 (1999).

${ }^{17}$ M. I. Marqués, J. A. Gonzalo, and J. Íñiguez, Phys. Rev. E 62, 191 (2000)

${ }^{18}$ V. V. Prudnikov, P. V. Prudnikov, and A. A. Fedorenko, Phys. Rev. B 62, 8777 (2000).

${ }^{19}$ A. P. Y. Wong and M. H. W. Chan, Phys. Rev. Lett. 65, 2567 (1990).

${ }^{20}$ A. P. Y. Wong, S. B. Kim, W. I. Goldburg, and M. H. W. Chan, Phys. Rev. Lett. 70, 954 (1993).

${ }^{21}$ A. Hasmy and R. Jullien, Phys. Rev. E 53, 1789 (1996).

${ }^{22}$ R. Guida and J. Zinn-Justin, J. Phys. A 31, 8103 (1998).

${ }^{23}$ H. G. Ballesteros, L. A. Fernández, V. Martín-Mayor, A. Muñoz Sudupe, G. Parisi, and J. J. Ruiz-Lorenzo, Phys. Rev. B 58, 2740 (1998).

${ }^{24}$ K. E. Newman and E. K. Riedel, Phys. Rev. B 25, 264 (1982).

${ }^{25}$ G. Jug, Phys. Rev. B 27, R609 (1983). 
${ }^{26}$ I. O. Mayer, J. Phys. A 22, 2815 (1989).

${ }^{27}$ J. C. Le Guillou and J. Zinn-Justin, Phys. Rev. B 21, 3976 (1980).

${ }^{28}$ P. Calabrese, P. Parruccini, A. Pelissetto, and E. Vicari, Phys. Rev. E 69, 036120 (2004).

${ }^{29}$ D. P. Landau, Phys. Rev. B 22, 2450 (1980).

${ }^{30}$ H. O. Heuer, Europhys. Lett. 12, 551 (1990)

${ }^{31}$ H. O. Heuer, Phys. Rev. B 42, 6476 (1990).

${ }^{32}$ P. E. Berche, C. Chatelain, B. Berche, and W. Janke, Europhys. Lett. 38, 463 (2004).

${ }^{33}$ D. P. Belanger, A. R. King, and V. Jaccarino, Phys. Rev. B 34, 452 (1986)

${ }^{34}$ P. W. Mitchell, R. A. Cowley, H. Yoshizawa, P. Böni, Y. J. Uemura, and R. J. Birgeneau, Phys. Rev. B 34, 4719 (1986).

${ }^{35}$ A. Hasmy, M. Foret, J. Pelous, and R. Jullien, Phys. Rev. B 48, 9345 (1993).

${ }^{36}$ A. Hasmy, E. Anglaret, M. Foret, J. Pelous, and R. Jullien, Phys. Rev. B 50, 6006 (1994); A. Hasmy and R. Jullien, J. Non-Cryst. Solids 186, 342 (1995).

${ }^{37}$ U. Wolff, Phys. Rev. Lett. 62, 361 (1989).

${ }^{38}$ A. M. Ferrenberg and D. P. Landau, Phys. Rev. B 44, 5081
(1991).

${ }^{39}$ A. M. Ferrenberg and R. H. Swendsen, Phys. Rev. Lett. 61, 2635 (1988).

${ }^{40}$ M. I. Marqués and J. A. Gonzalo, Phys. Rev. E 65, 057104 (2002).

${ }^{41}$ S. Wiseman and E. Domany, Phys. Rev. E 52, 3469 (1995); Phys. Rev. Lett. 81, 22 (1998).

${ }^{42}$ S. Wiseman and E. Domany, Phys. Rev. E 58, 2938 (1998).

${ }^{43}$ A. Aharony and A. B. Harris, Phys. Rev. Lett. 77, 3700 (1996).

${ }^{44}$ M. E. Fisher and M. N. Barber, Phys. Rev. Lett. 28, 1516 (1972); M. E. Fisher, Rev. Mod. Phys. 46, 597 (1972).

${ }^{45}$ K. Binder, Phys. Rev. Lett. 47, 693 (1981).

${ }^{46}$ N. Schultka and E. Manousakis, Phys. Rev. B 52, 7528 (1995).

${ }^{47}$ U. Wolff, Nucl. Phys. B 334, 581 (1990).

${ }^{48}$ D. V. Pakhnin and A. I. Sokolov, Phys. Rev. B 61, 15130 (2000).

${ }^{49}$ Manuel Marqués (private communication).

${ }^{50}$ Y. K. Wu and B. Hu, Phys. Rev. A 35, 1404 (1987); Yu. Holovatch and T. Krokhmal's'kii, J. Math. Phys. 35, 3866 (1994); V. Blavats'ka, C. von Ferber, and Yu. Holovatch, Phys. Rev. E 64, 041102 (2004). 